\title{
Serum Fibroblast Growth Factor 23 (FGF23) in Patients with Rheumatoid Arthritis
}

\author{
Hiroe Sato ${ }^{1}$, Junichiro James Kazama ${ }^{2}$, Akira Murasawa ${ }^{1}$, Hiroshi Otani ${ }^{1}$, Asami Abe ${ }^{1}$, \\ Satoshi Ito ${ }^{1}$, Hajime Ishikawa ${ }^{1}$, Kiyoshi Nakazono ${ }^{1}$, Takeshi Kuroda ${ }^{3}$, \\ Masaaki Nakano ${ }^{4}$ and Ichiei Narita ${ }^{2}$
}

\begin{abstract}
Objective Rheumatoid arthritis (RA) is a chronic inflammatory disease accompanied by periarticular and systemic osteoporosis. Fibroblast growth factor 23 (FGF23), which is mainly produced by osteocytes, circulates to the kidneys and regulates bone metabolism. We herein assessed serum FGF23 and its relationship to inflammation and osteoporosis in patients with RA.

Methods Sixty-one patients with RA were included. Serum concentrations of FGF23 were determined using a sandwich enzyme-linked immunosorbent assay.

Results The mean ( \pm standard deviation) serum FGF23 concentration was 34.9 \pm 9.2 (range, 21.0-61.0) pg/ $\mathrm{mL}$. The serum FGF23 level was significantly and positively correlated with the erythrocyte sedimentation rate (ESR), C-reactive protein (CRP) levels, disease activity score-28 based on the ESR (DAS-28 ESR) and DAS-28 CRP ( $\mathrm{r}=0.261, \mathrm{p}=0.044, \mathrm{r}=0.280, \mathrm{p}=0.029, \mathrm{r}=0.409, \mathrm{p}=0.001$ and $\mathrm{r}=0.421, \mathrm{p}=0.001$, respectively). The serum matrix metalloproteinase-3 level was also significantly and positively correlated with the serum FGF23 level $(r=0.331, p=0.015)$. Concentrations of type I collagen cross-linked $\mathrm{N}$-telopeptide in the serum was significantly correlated with the serum FGF23 level $(r=0.272, p=0.034)$. Neither the bone mineral density in the femoral neck nor lumbar was significantly correlated with the serum FGF23 level. Serum phosphate, calcium, 25-hydroxy vitamin D, and intact parathyroid hormone were not related to the serum FGF23 level. Conclusion In patients with RA, serum FGF23 is correlated with inflammation, the disease activity of RA, and bone absorption markers. Serum FGF23 may be associated with abnormal bone absorption related to RA inflammation. Further studies are necessary to clarify the mechanism underlying this association.
\end{abstract}

Key words: fibroblast growth factor 23 , inflammation, osteoporosis, rheumatoid arthritis

(Intern Med 55: 121-126, 2016)

(DOI: 10.2169/internalmedicine.55.5507)

\section{Introduction}

Rheumatoid arthritis (RA) is an inflammatory disease that mainly affects the synovial tissue. Inflammatory cytokines, such as interleukin (IL)-1, IL-6, and tumor necrosis factor (TNF), activate osteoclasts and cause periarticular bone atrophy and destruction in patients with RA $(1,2)$. In addition to periarticular osteoporosis, systemic osteoporosis is often associated with RA; several mechanisms have been proposed to underlie the increased risk for osteoporosis in patients with RA, including RA-related inflammation, glucocorticoid treatment, and immobility due to arthralgia or joint deformity.

Fibroblast growth factor 23 (FGF23), which is secreted mainly by osteocytes, circulates to the kidneys, where it decreases phosphate reabsorption and inhibits 25-hydroxy vitamin $\mathrm{D}_{3}$ 1-alpha-hydroxylase, causing decreased circulating

\footnotetext{
${ }^{1}$ Department of Rheumatology, Niigata Rheumatic Center, Japan, ${ }^{2}$ Division of Clinical Nephrology and Rheumatology, Niigata University Graduate School of Medical and Dental Sciences, Japan, ${ }^{3}$ Niigata University Health Administration Center, Japan and ${ }^{4}$ Department of Medical Technology, School of Health Sciences, Faculty of Medicine, Niigata University, Japan

Received for publication April 3, 2015; Accepted for publication June 16, 2015

Correspondence to Dr. Hiroe Sato, hiroe-212@umin.ac.jp
} 
1,25-dihydroxy vitamin $\mathrm{D}_{3}\left[1,25(\mathrm{OH})_{2} \mathrm{D}_{3}\right]$ (3-5). Diets high in phosphate raise the serum FGF23 levels in healthy subjects $(6)$, as does the administration of $1,25(\mathrm{OH})_{2} \mathrm{D}_{3}$ in animals $(7,8)$.

FGF23-null mice are characterized by severe hyperphosphatemia, hypervitaminosis $\mathrm{D}$, hypercalcemia, early-onset osteomalacia, growth retardation, and a short lifespan $(9,10)$. On the other hand, mice overexpressing FGF23 display hypophosphatemia, low $1,25(\mathrm{OH})_{2} \mathrm{D}_{3}$ levels, and markedly decreased bone mineral content (11). Thus, abnormal FGF23 expression influences bone mineralization via alterations in phosphate and $1,25(\mathrm{OH})_{2} \mathrm{D}_{3}$ metabolism. The purpose of this study was to elucidate whether serum FGF23 is related to RA-associated bone destruction and systemic osteoporosis.

\section{Materials and Methods}

\section{Subjects}

The study subjects comprised 61 women with RA treated at the Niigata Rheumatic Center between May 2008 and October 2010. All patients included in this study had normal serum creatinine $(\mathrm{Cr})$ levels $(\leq 0.8 \mathrm{mg} / \mathrm{dL})$ and met the 1987 American Rheumatism Association criteria for RA (12). The anatomical stage and functional class were determined using the system proposed by Steinbrocker et al. (13).

The study protocol was approved by the ethics committee of the Niigata Rheumatic Center and executed according to the Declaration of Helsinki.

\section{Calculation of the estimated glomerular filtration rate}

Estimated glomerular filtration rates (eGFRs) were calculated using the standard formula for women, $194 \times \mathrm{Cr}^{-1.094} \times$ Age $^{-0.287} \times 0.739$, which was developed using the inulin clearance of Japanese subjects as a standard (14).

\section{Biochemical assays of FGF23 and other bone me- tabolism markers}

FGF23 concentrations were determined using serum samples collected and stored at $-80^{\circ} \mathrm{C}$ with a sandwich enzymelinked immunosorbent assay (Kainos Laboratories, Tokyo, Japan), which detected full-length human FGF23 (15). Serum and urine concentrations of type I collagen cross-linked N-telopeptide (NTx) were measured and urine NTx (uNTx) was normalized to urine $\mathrm{Cr}$. Undercarboxylated osteocalcin (ucOC) and intact parathyroid hormone (PTH) were measured by an electrochemiluminescent immunoassay (EIDIA, Tokyo, Japan and Roche Diagnostics, Tokyo, Japan, respectively). Tartrate-resistant acid phosphatase-5b (TRACP-5b) was measured using an enzyme immunoassay (Nittobo Medical, Tokyo, Japan). 25-hydroxy vitamin D [25(OH)D] and quantified by a radioimmunoassay (Diasorin, Stillwater, USA).

The normal ranges of the bone metabolism markers were as follows: alkaline phosphatase, $115-359 \mathrm{U} / \mathrm{L}$; bone alkaline phosphatase, $2.9-14.5 \mu \mathrm{g} / \mathrm{L}$ for premenopausal women and 3.8-22.6 $\mu \mathrm{g} / \mathrm{L}$ for postmenopausal women; ucOC, $<4.50$ $\mathrm{ng} / \mathrm{mL}$; serum NTx, 7.5-16.5 nmol BCE/L for premenopausal women and 10.7-24.0 nmol $\mathrm{BCE} / \mathrm{L}$ for postmenopausal women; urine NTx, 9.3-54.3 $\mathrm{nmol} \mathrm{BCE} / \mathrm{mmol} \cdot \mathrm{Cr}$ for premenopausal women and 14.3-89.0 nmol BCE/mmol $\cdot \mathrm{Cr}$ for postmenopausal women; TRACP-5b, 120-420 mU/dL for women; 25(OH)D, 7-41 ng/mL; and intact PTH, 10-65 pg/ $\mathrm{mL}$.

\section{Bone mineral density measurement}

The bone mineral density (BMD) was measured using dual-energy X-ray absorptiometry. The BMD was measured twice in each participant at the time of enrollment and 1 year later.

\section{Statistical analysis}

Most data are expressed as the means \pm standard deviations. The relationships between FGF23 and other markers were analyzed using Pearson's correlation coefficients when the distributions were normal using Kolmogorov-Smirnov test, and Spearman's rank correlation coefficients were used when the distributions were not normal. Partial correlation coefficients were used to assess age and the eGFR adjusted associations between serum FGF23 and renal markers.

All statistical analyses were performed with the SPSS software program (ver. 19, SPSS, Chicago, USA). $\mathrm{p}<0.05$ was considered to be statistically significant.

\section{Results}

\section{Characteristics of the study subjects}

The characteristics of the study subjects are shown in Table 1 . The mean duration of RA was $13.7 \pm 11.8$ years. The mean disease activity scores in 28 joints (DAS28) according to the serum C-reactive protein (CRP) and erythrocyte sedimentation rate (ESR) indicated moderate disease activity $(4.0 \pm 1.4$ and $4.7 \pm 1.4$, respectively). Only patients with RA whose serum $\mathrm{Cr}$ was within the normal range were included; the mean serum $\mathrm{Cr}$ concentration was low $(0.56 \pm$ $0.12 \mathrm{mg} / \mathrm{dL})$. However, the mean eGFR was $49.3 \pm 3.5 \mathrm{~mL} /$ $\min / 1.73 \mathrm{~m}^{2}$, indicating stage 3 chronic kidney disease (CKD) and a moderate reduction in the kidney function.

\section{Mean concentrations of FGF23 and other bone me- tabolism markers}

The mean serum FGF23 concentration was 34.9 \pm 9.2 (range, 21.0-61.0) $\mathrm{pg} / \mathrm{mL}$. The mean serum concentrations of alkaline phosphatase (ALP), bone-specific alkaline phosphatase, ucOC, NTx, uNTx, and TRACP-5b were all within the normal ranges for women (Table 2). 
Table 1. Characteristics of Patients with Rheumatoid Arthritis.

\begin{tabular}{crl}
\hline & Mean & $\pm \mathrm{SD}$ \\
\hline Age, years & 63.6 & \pm 9.9 \\
Height, cm & 149.6 & \pm 7.1 \\
Body weight, kg & 50.6 & \pm 9.4 \\
Body mass index, kg/m² & 22.5 & \pm 3.4 \\
Steinbrocker stage & $\mathrm{I}, 1 ; \mathrm{II}, 15 ; \mathrm{III}, 18 ; \mathrm{IV}, 27$ \\
Steinbrocker class & 1,$2 ; 2,37 ; 3,22 ; 4,0$ \\
Duration of RA, years & 13.7 & \pm 11.8 \\
Prednisolone, mg/day & 3.3 & \pm 2.7 \\
Methotrexate, mg/week & 4.0 & \pm 3.6 \\
Biological agent use, $\mathrm{n}(\%)$ & 4 & $(6.6)$ \\
C-reactive protein, mg/dL & 3.2 & \pm 3.4 \\
Hemoglobin & 11.6 & \pm 1.6 \\
Drythrocyte sedimentation rate, mm/h & 52.4 & \pm 35.0 \\
DAS28-ESR & 4.7 & \pm 1.4 \\
DAS28-CRP & 4.0 & \pm 1.4 \\
MMP-3 & 279.3 & \pm 283.0 \\
Serum creatinine, mg/dL & 0.56 & \pm 0.12 \\
eGFR, mL/min & 49.3 & \pm 3.5 \\
\hline
\end{tabular}

SD: standard deviation, RA: rheumatoid arthritis, DAS: disease activity score, ESR: erythrocyte sedimentation rate, CRP: C-reactive protein, MMP-3: matrix metalloproteinase-3, eGFR: estimated glomerular filtration rate
Table 2. Serum Fibroblast Growth Factor 23, Markers of Bone Metabolism, and Drugs for Osteoporosis.

\begin{tabular}{lrl}
\hline & mean & $\pm \mathrm{SD}$ \\
\hline FGF23, pg/mL & 34.9 & \pm 9.2 \\
Serum phosphate, mg/dL & 3.5 & \pm 0.5 \\
Serum calcium, mg/dL & 9.5 & \pm 0.4 \\
Serum phosphate $\times$ serum calcium, mg2/dL 2 & 32.8 & \pm 5.6 \\
ALP, U/L & 281.0 & \pm 120.8 \\
Bone ALP, U/L & 21.1 & \pm 9.9 \\
ucOC, ng/mL & 3.5 & \pm 2.7 \\
NTx, nmolBCE/L & 18.2 & \pm 6.0 \\
uNTx, nmolBCE/nmol • Cr & 58.2 & \pm 29.5 \\
TRACP-5b, mU/dL & 304.8 & \pm 156.2 \\
25(OH)D, ng/mL & 26.4 & \pm 8.8 \\
Intact PTH, pg/mL & 25.2 & \pm 13.4 \\
Bisphosphonate use, $\mathrm{n}(\%)$ & 22 & $(36.1)$ \\
Active vitamin D use, $\mathrm{n}(\%)$ & 3 & $(4.9)$ \\
Calcium preparation use, $\mathrm{n}(\%)$ & 0 & $(0)$ \\
Vitamin K ${ }_{2}$ use, $\mathrm{n}(\%)$ & 1 & $(1.6)$ \\
Selective estrogen receptor modulator, $\mathrm{n}(\%)$ & 0 & $(0)$ \\
\hline
\end{tabular}

SD: standard deviation, FGF23: fibroblast growth factor 23, ALP: alkaline phosphatase, ucOC: undercarboxylated osteocalcin, NTx: serum type I collagen cross-linked N-telopeptide, uNTx: urine type I collagen cross-linked N-telopeptide, Cr: creatinine, TRACP-5b: tartrate-resistant acid phosphatase-5b, 25(OH)D: 25-hydroxy vitamin D, PTH: parathyroid hormone

the FGF23 levels in healthy controls, according to a previous report regarding full-length FGF23, the average serum FGF23 concentration in healthy individuals is $28.9 \pm 1.1$ (range, 8.2-54.3) pg/mL (15). The FGF23 levels measured

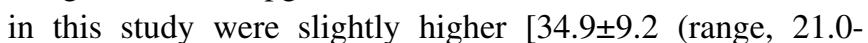
61.0) $\mathrm{pg} / \mathrm{mL}$. However, the measurement method of that study differed from that used in the present study and the value served only as a reference. The serum FGF23 concentrations are higher than normal in patients with renal insufficiency (16) and markedly elevated in patients on dialysis (17-19). Although this study included only patients with serum $\mathrm{Cr}$ concentrations within the normal range, the mean eGFR indicated stage $3 \mathrm{CKD}$ and a moderate reduction in the GFR $\left(30-59 \mathrm{~mL} / \mathrm{min} / 1.73 \mathrm{~m}^{2}\right)$. This reduction likely results from decreased muscle mass, as patients with RA are generally hypoactive, and serum $\mathrm{Cr}$ levels correlate with muscle mass (20). Although the accuracy of the eGFR as an indicator of kidney function has not been validated in patients with low muscle mass, such as those with RA, serum FGF23 did not appear to be related to serum phosphate, calcium, 25(OH)D, or intact PTH in this study, suggesting that FGF23 is unrelated to the kidney function.

The disease activity of RA and inflammatory markers (CRP and ESR) were significantly related to serum FGF23 in this study. Among patients with CKD ( $n=3,879$; eGFR, 20-70 $\mathrm{mL} / \mathrm{min} / 1.73 \mathrm{~m}^{2}$; age, 21-74 years), FGF23 and inflammatory markers (CRP, IL-6, TNF, and fibrinogen) were positively correlated and FGF23 concentrations were correlated with the severity of inflammation (21). Another study including 145 patients with CKD stages 1-4 found that serum FGF23 was related to serum CRP but not phosphate, and that FGF23 concentrations were related to the BMD in 
Table 3. Correlations between Fibroblast Growth Factor 23 and Study Parameters.

\begin{tabular}{|c|c|c|}
\hline & Correlation coefficient & $\mathrm{p}$ \\
\hline Age & 0.084 & 0.519 \\
\hline Height & -0.074 & 0.571 \\
\hline Body weight & -0.043 & 0.744 \\
\hline Body mass index & -0.018 & 0.891 \\
\hline $\begin{array}{l}\text { Disease duration of rheumatoid } \\
\text { arthritis }\end{array}$ & -0.057 & 0.663 \\
\hline Methotrexate dose & 0.198 & 0.127 \\
\hline Prednisolone dose & 0.156 & 0.231 \\
\hline Duration of prednisolone usage & 0.079 & 0.547 \\
\hline Biological agent use & -0.040 & 0.758 \\
\hline DAS28-ESR & $0.409^{*}$ & 0.001 \\
\hline DAS28-CRP & $0.421^{*}$ & 0.001 \\
\hline C-reactive protein & $0.280^{*}$ & 0.029 \\
\hline Erythrocyte sedimentation rate & $0.261^{*}$ & 0.044 \\
\hline MMP-3 & $0.331^{*}$ & 0.015 \\
\hline Serum creatinine & 0.049 & 0.708 \\
\hline eGFR & -0.107 & 0.412 \\
\hline Serum albumin & -0.222 & 0.086 \\
\hline Hemoglobin & -0.113 & 0.387 \\
\hline Femoral neck BMD & -0.234 & 0.072 \\
\hline Change per year of femoral neck BMD & -0.239 & 0.069 \\
\hline Lumbar BMD & -0.085 & 0.515 \\
\hline Change per year of lumbar BMD & 0.045 & 0.732 \\
\hline Serum phosphate & -0.250 & 0.054 \\
\hline Serum calcium & 0.180 & 0.168 \\
\hline Serum phosphate $\times$ serum calcium & -0.175 & 0.180 \\
\hline Osteogenesis ALP & 0.222 & 0.085 \\
\hline Bone ALP & -0.017 & 0.895 \\
\hline ucOC & 0.110 & 0.399 \\
\hline TRACP-5b & -0.067 & 0.609 \\
\hline absorption & $0.272^{*}$ & 0.034 \\
\hline markers & 0.232 & 0.072 \\
\hline $25(\mathrm{OH}) \mathrm{D}$ & -0.028 & 0.837 \\
\hline Intact PTH & -0.057 & 0.675 \\
\hline \multicolumn{3}{|c|}{$\begin{array}{l}\text { DAS: disease activity score, ESR: erythrocyte sedimentation rate, CRP } \\
\text { C-reactive protein, MMP-3: matrix metalloproteinase-3, eGFR: estimated } \\
\text { glomerular filtration rate, BMD: bone mineral density, ALP: alkaline } \\
\text { phosphatase, ucOC: undercarboxylated osteocalcin, TRACP-5b } \\
\text { tartrate-resistant acid phosphatase-5b, NTx: serum type I collagen } \\
\text { cross-linked N-telopeptide, uNTx: urine type I collagen cross-linked } \\
\text { N-telopeptide, 25(OH)D: 25-hydroxy vitamin D, PTH: parathyroic } \\
\text { hormone }\end{array}$} \\
\hline
\end{tabular}

Table 4. Partial Correlation Coefficients Controlled for Age and eGFR between Fibroblast Growth Factor 23 and Renal Parameters.

\begin{tabular}{lcc}
\hline & Partial correlation coefficient & $\mathrm{p}$ \\
Serum phosphate & -0.238 & 0.072 \\
Serum calcium & 0.217 & 0.101 \\
Serum phosphate $\times$ serum calcium & -0.161 & 0.228 \\
25(OH)D & -0.046 & 0.739 \\
Intact PTH & 0.030 & 0.814 \\
\hline
\end{tabular}

25(OH)D: 25-hydroxy vitamin D, PTH: parathyroid hormone

the total hip (22). As the cells that produce FGF23 and the stimulus that triggers FGF23 production have not yet been identified, the mechanism linking inflammation and serum FGF23 has not yet been elucidated. The disordered iron metabolism associated with chronic inflammation may be the link between FGF23 and inflammation. Under inflammatory conditions, elevated IL-6 increases the hepatic production of hepcidin, which inhibits iron absorption from the intestines and blocks iron release from macrophages and hepatocytes (23). Iron deficiency anemia is associated with high levels of FGF23, which decrease with the administration of intravenous elemental iron (23). Thus, lower circulating iron levels and/or higher ferritin levels resulting from chronic inflammation could increase serum FGF23. Indeed, Braith- 
waite et al. (24) reported that serum FGF23 was more closely related to ferritin than to CRP. In this study, the hemoglobin level was not significantly correlated with FGF23 and the serum iron and ferritin levels were not examined. Further studies are warranted to reveal the relationship between iron metabolism and FGF23.

Our results indicate a possible association between RArelated bone destruction and FGF23, because MMP-3 and DAS were significantly related to FGF23. Although FGF23 is produced by osteocytes, its concentration in the serum may not reflect the total number of viable osteocytes. In elderly patients with pre-dialysis CKD, the risk of vertebral fractures was positively associated with the serum FGF23 levels, and the elevation of FGF23 concentrations was independently correlated with osteoporosis (25). Patients with hip fractures have more apoptotic osteocytes and lower RNA transcription of FGF23 than controls (26). In the same report, patients with osteoarthritis had lower than normal expression of FGF23 and normal levels of osteocyte apoptosis. In our study, FGF23 was not significantly related to the $\mathrm{BMD}$; however, there was a tendency of a negative relationship between FGF23 and the femoral neck BMD and its annual rate of change $(\mathrm{r}=-0.234, \mathrm{p}=0.072$ and $\mathrm{r}=-0.239, \mathrm{p}=$ 0.069 , respectively) (Table 3). Although the lumbar BMD was not significantly related to the FGF23 levels, it is less accurate as a marker for osteoporosis than the femoral neck BMD due to a compression fracture or deformity. Studies with a greater accumulation of patients are necessary to determine the influence of serum FGF23 levels in systemic osteoporosis in patients with RA.

A relationship between bone absorption and FGF23 has also been previously reported. Kitaoka et al. (27) reported a rapid decrease in FGF23 and NTx following pamidronate infusion in patients with osteogenesis imperfecta, indicating that FGF23 could be closely related to bone resorption. In this study, NTx was significantly correlated with serum FGF23. NTx is generally increased after menopause in association with age-related bone loss, however, FGF23 was not related to age in this study and age and sex-dependent differences in the serum FGF23 level were not reported in a previous study (15). The FGF23 and NTx levels in patients with RA appear to be more strongly influenced by inflammatory bone absorption associated with RA than with agerelated bone loss. Taken together, these findings suggest that RA-related inflammation influences the serum FGF23 levels and that periarticular or systemic bone resorption is influenced by the serum FGF23 levels in patients with RA.

FGF23 can directly affect bone metabolism independently of phosphate metabolism. FGF23 is expressed by osteocytes and osteoblasts, and its receptors are expressed by osteoblasts $(28,29)$; thus, FGF23 could mediate signaling to osteoblasts. The overexpression of FGF23 during osteoblast development in fetal rat calvaria cell cultures suppressed osteoblast differentiation and matrix mineralization independently of phosphate homeostasis (29). In animal studies, FGF 23 -induced demineralization was too rapid to involve renal tubular phosphate metabolism (30). As discussed above, several reports have indicated a direct influence of FGF23 on bone metabolism. However, the mechanism by which RA alters FGF23 concentrations has not yet been elucidated and further basic studies are therefore warranted.

A major limitation associated with this study was that we were only able to measure $25(\mathrm{OH}) \mathrm{D}$, not $1,25(\mathrm{OH})_{2} \mathrm{D}_{3}$. Thus, the possibility remains that $1,25(\mathrm{OH})_{2} \mathrm{D}_{3}$ links FGF23 to bone destruction and osteoporosis, which warrants investigation. Furthermore, sclerostin is a humoral factor that strongly inhibits bone formation (31) and elevated circulating levels of this factor are associated with the incidence of osteoporosis in postmenopausal women (32). Because sclerostin is also released from osteocytes (33), its net function may be indicated by circulating FGF23 levels, which may in turn be affected by osteocyte activity. However, we were unable to monitor the circulating sclerostin levels in this study. Additionally, joint destruction using the modified Sharp score or ultrasonography was not evaluated, and the relationship between sequential joint destruction and the FGF23 levels remains poorly defined.

\section{Conclusion}

In conclusion, the serum FGF23 levels in patients with RA were positively correlated with inflammation, the disease activity of RA, and bone resorption markers. These results indicate that abnormal bone absorption related to RA inflammation may affect the serum FGF23 level in RA. Further studies are necessary to clarify the mechanism of this association.

The authors state that they have no Conflict of Interest (COI).

\section{References}

1. Edwards CJ, Williams E. The role of interleukin-6 in rheumatoid arthritis-associated osteoporosis. Osteoporos Int 21: 1287-1293, 2010.

2. Schett G, Saag KG, Bijlsma JW. From bone biology to clinical outcome: state of the art and future perspectives. Ann Rheum Dis 69: 1415-1419, 2010.

3. Liu S, Zhou J, Tang W, Jiang X, Rowe DW, Quarles LD. Pathogenic role of Fgf23 in Hyp mice. Am J Physiol Endocrinol Metab 291: E38-E49, 2006.

4. Shimada T, Hasegawa H, Yamazaki Y, et al. FGF-23 is a potent regulator of vitamin D metabolism and phosphate homeostasis. J Bone Miner Res 19: 429-435, 2004.

5. Shimada T, Yamazaki Y, Takahashi M, et al. Vitamin D receptorindependent FGF23 actions in regulating phosphate and vitamin D metabolism. Am J Physiol Renal Physiol 289: F1088-F1095, 2005.

6. Nishida Y, Taketani Y, Yamanaka-Okumura H, et al. Acute effect of oral phosphate loading on serum fibroblast growth factor 23 levels in healthy men. Kidney Int 70: 2141-2147, 2006.

7. Kolek OI, Hines ER, Jones MD, et al. 1 $\alpha, 25$-Dihydroxyvitamin $\mathrm{D}_{3}$ upregulates FGF23 gene expression in bone: the final link in a renal-gastrointestinal-skeletal axis that controls phosphate transport. Am J Physiol Gastrointest Liver Physiol 289: G1036-G1042, 
2005.

8. Saito H, Maeda A, Ohtomo S, et al. Circulating FGF-23 is regulated by $1 \alpha, 25$-dihydroxyvitamin $\mathrm{D}_{3}$ and phosphorus in vivo. $\mathrm{J}$ Biol Chem 280: 2543-2549, 2005.

9. Sitara D, Kim S, Razzaque MS, et al. Genetic evidence of serum phosphate-independent functions of FGF-23 on bone. PLoS Genet 4: e1000154, 2008.

10. Sitara D, Razzaque MS, Hesse M, et al. Homozygous ablation of fibroblast growth factor-23 results in hyperphosphatemia and impaired skeletogenesis, and reverses hypophosphatemia in Phexdeficient mice. Matrix Biol 23: 421-432, 2004.

11. Shimada T, Mizutani S, Muto T, et al. Cloning and characterization of FGF23 as a causative factor of tumor-induced osteomalacia. Proc Natl Acad Sci USA 98: 6500-6505, 2001.

12. Arnett FC, Edworthy SM, Bloch DA, et al. The American Rheumatism Association 1987 revised criteria for the classification of rheumatoid arthritis. Arthritis Rheum 31: 315-324, 1988.

13. Steinbrocker $\mathrm{O}$, Traeger $\mathrm{CH}$, Batterman RC. Therapeutic criteria in rheumatoid arthritis. J Am Med Assoc 140: 659-662, 1949.

14. Matsuo $S$, Imai E, Horio $M$, et al. Revised equations for estimated GFR from serum creatinine in Japan. Am J Kidney Dis 53: 982992, 2009.

15. Yamazaki Y, Okazaki R, Shibata M, et al. Increased circulatory level of biologically active full-length FGF-23 in patients with hypophosphatemic rickets/osteomalacia. J Clin Endocrinol Metab 87: 4957-4960, 2002

16. Isakova T, Wahl P, Vargas GS, et al. Fibroblast growth factor 23 is elevated before parathyroid hormone and phosphate in chronic kidney disease. Kidney Int 79: 1370-1378, 2011.

17. Isakova $\mathrm{T}, \mathrm{Xie} \mathrm{H}$, Barchi-Chung A, et al. Fibroblast growth factor 23 in patients undergoing peritoneal dialysis. Clin J Am Soc Nephrol 6: 2688-2695, 2011.

18. Park SY, Jeong KH, Moon JY, et al. The relationship between circulating fibroblast growth factor 23 and bone metabolism factors in Korean hemodialysis patients. Clin Exp Nephrol 14: 239-243, 2010.

19. Urena Torres P, Friedlander G, de Vernejoul MC, Silve C, Prie D. Bone mass does not correlate with the serum fibroblast growth factor 23 in hemodialysis patients. Kidney Int 73: 102-107, 2008.

20. Sato H, Kuroda $T$, Tanabe $N$, et al. Cystatin $C$ is a sensitive marker for detecting a reduced glomerular filtration rate when assessing chronic kidney disease in patients with rheumatoid arthritis and secondary amyloidosis. Scand J Rheumatol 39: 33-37, 2010.
21. Munoz Mendoza J, Isakova $T$, Ricardo $A C$, et al. Fibroblast growth factor 23 and inflammation in CKD. Clin J Am Soc Nephrol 7: 1155-1162, 2012.

22. Manghat P, Fraser WD, Wierzbicki AS, Fogelman I, Goldsmith DJ, Hampson G. Fibroblast growth factor-23 is associated with Creactive protein, serum phosphate and bone mineral density in chronic kidney disease. Osteoporos Int 21: 1853-1861, 2010.

23. Wolf M, Koch TA, Bregman DB. Effects of iron deficiency anemia and its treatment on fibroblast growth factor 23 and phosphate homeostasis in women. J Bone Miner Res 28: 1793-1803, 2013.

24. Braithwaite V, Prentice AM, Doherty C, Prentice A. FGF23 is correlated with iron status but not with inflammation and decreases after iron supplementation: a supplementation study. Int J Pediatr Endocrinol 2012: 27, 2012.

25. Kanda E, Yoshida M, Sasaki S. Applicability of fibroblast growth factor 23 for evaluation of risk of vertebral fracture and chronic kidney disease-mineral bone disease in elderly chronic kidney disease patients. BMC Nephrol 13: 122, 2012.

26. Delgado-Calle J, Arozamena J, Garcia-Renedo R, et al. Osteocyte deficiency in hip fractures. Calcif Tissue Int 89: 327-334, 2011.

27. Kitaoka T, Namba N, Miura K, et al. Decrease in serum FGF23 levels after intravenous infusion of pamidronate in patients with osteogenesis imperfecta. J Bone Miner Metab 29: 598-605, 2011.

28. Rice DP, Aberg T, Chan Y, et al. Integration of FGF and TWIST in calvarial bone and suture development. Development 127: 1845-1855, 2000.

29. Wang H, Yoshiko Y, Yamamoto R, et al. Overexpression of fibroblast growth factor 23 suppresses osteoblast differentiation and matrix mineralization in vitro. J Bone Miner Res 23: 939-948, 2008.

30. Quarles LD. FGF23, PHEX, and MEPE regulation of phosphate homeostasis and skeletal mineralization. Am J Physiol Endocrinol Metab 285: E1-E9, 2003.

31. Moester MJ, Papapoulos SE, Lowik CW, van Bezooijen RL. Sclerostin: current knowledge and future perspectives. Calcif Tissue Int 87: 99-107, 2010.

32. Polyzos SA, Anastasilakis AD, Bratengeier C, Woloszczuk W, Papatheodorou A, Terpos E. Serum sclerostin levels positively correlate with lumbar spinal bone mineral density in postmenopausal women: the six-month effect of risedronate and teriparatide. Osteoporos Int 23: 1171-1176, 2012.

33. van Bezooijen RL, Roelen BA, Visser A, et al. Sclerostin is an osteocyte-expressed negative regulator of bone formation, but not a classical BMP antagonist. J Exp Med 199: 805-814, 2004.

(C) 2016 The Japanese Society of Internal Medicine http://www.naika.or.jp/imonline/index.html 